\title{
VARIABILIDAD ESPACIO-TEMPORAL DE CONDICIONES HIDROFÍSICAS EN BAHÍA INDEPENDENCIA, PISCO - PERÚ, DESDE 1995 AL 2004
}

\section{SPATIO-TEMPORAL VARIABILITY OF HYDROPHYSICAL CONDITIONS AT BAHIA INDEPENDENCIA, PISCO - PERÚ, FROM 1995 TO 2004}

\author{
Daniel Quispe ${ }^{1}$, Michelle Graco ${ }^{2}$, David Correa ${ }^{3}$, Jorge Tam ${ }^{4}$, \\ Dimitri Gutiérrez ${ }^{5}$, Octavio Morón ${ }^{6}$, Georgina Flores ${ }^{7}$ y Carmen Yamashiro ${ }^{8}$
}

\begin{abstract}
Resumen
Bahía Independencia cuenta con un importante sistema marino cuya estructura y funcionalidad, ha sido objeto de diferentes estudios biológicos. A partir de informes de evaluaciones in situ (IMARPE, DHN) y de datos satelitales (QuikSCAT), se logró describir la variabilidad espacio-temporal de las condiciones hidrofísicas, en base a vientos, circulación marina, temperatura y salinidad. Para ello se aplicó análisis de estadística descriptiva, pruebas de correlación, análisis multivariado, análisis de varianza (ANOVA) y mapas con escalas de color.

Se identificaron patrones de distribución espacial cuya formación se atribuye a factores locales. En superficie se manifiesta un gradiente latitudinal de temperatura y en menor proporción de salinidad, con valores altos al norte de la bahía, debido al repliegue de aguas por acción del viento y a la influencia de Laguna Grande, una formación semi-encerrada en el extremo norte de la bahía. A nivel de fondo, en la zona norte la distribución de temperatura y salinidad reflejan el proceso de afloramiento, evidenciado por aguas que circulan de manera transversal al eje mayor de la bahía. En cambio, en la zona sur la circulación logra acoplarse en ambos niveles de profundidad con flujos en sentido horario y antihorario, generando pequeñas zonas con características similares de salinidad. A la bahía incursionan con mayor frecuencia las Aguas Ecuatoriales Subsuperficiales (AESS) afloradas, en algunos períodos aguas superficiales de mezcla con influencia de las aguas subtropicales superficiales (ASS) y en condiciones extremas El Niño, como en 1998, las ASS, más cálidas y salinas.
\end{abstract}

Palabras clave: oceanografía, temperatura, salinidad, Bahía Independencia, Perú

\begin{abstract}
Bahía Independencia (Independence Bay) is a marine system whose structure and function, have been subject of various biological studies. From in situ assessment reports (IMARPE, DHN) and satellite data (QuikSCAT), variations in spatio-temporal hydrophysical conditions, based on winds, ocean circulation, temperature and salinity were described. For the analysis, descriptive statistics, correlation tests, multivariate analysis, analysis of variance (ANOVA) and maps with color scales were applied.

Spatial distribution patterns whose formation is attributed to local factors were identified. On the surface there are latitudinal gradients of temperature and of salinity to a lesser extent, with higher values in the north of the bay, because of water drift due to wind stress and the influence of Laguna Grande, a semi-enclosed formation at the northern end of the Bay. Near the bottom level, in the north, temperature and salinity distributions reflect the upwelling circulation, evidenced by circulating water transversely to the long axis of the bay. Conversely, in the south, surface and near bottom circulations were coupled, determining clockwise and counter-clockwise fluxes, creating small areas with similar characteristics of salinity. The bay is normally intruded by upwelling Equatorial Subsurface Waters, and sometimes by mixed waters with Subtropical Surface Waters (ESSW). In the extreme 1997-98 El Niño the SSW occupied all the bay area.
\end{abstract}

Key words: oceanography, temperature, salinity, Bahía Independencia, Perú

\section{Introducción.}

El sistema marino de Bahía Independencia $\left(14^{\circ}\right.$

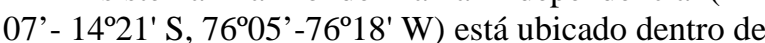
la Reserva Nacional de Paracas - Pisco - Perú y, es importante por su diversidad biológica, cuya composición, estructura y funcionalidad ha sido objeto de múltiples investigaciones.

La Figura 1 presenta un mapa batimétrico de Bahía Independencia y de sus puntos de referencia. El eje mayor de la bahía es de SE a NW. Entre Playa 
Ventosa y la boca de Laguna Grande mide aprox. 24.7 $\mathrm{km}$, desde el norte de la Isla Independencia hasta Punta Carhuas 6.8 km (eje menor). El área de toda la bahía es aprox. 15644.88 ha, Laguna Grande cubre 299.29 ha. La configuración batimétrica en la zona norte de la bahía proyecta un declive hacia el sur-oeste a través del Canal La Trujillana, que separa Punta Carretas y el norte de la Isla Independencia, alcanzando profundidades entre 80 a $90 \mathrm{~m}$, el ancho del canal es $8.95 \mathrm{~km}$. Este declive es limitado en el centro de la bahía por la Isla Independencia y una extensión de baja profundidad entre la isla y la costa central de la bahía conocida como La Pampa cuyas profundidades se hallan entre 10 a $20 \mathrm{~m}$. Desde ésta zona hacia el sur, la bahía presenta una extensa plataforma de entre 30 a $40 \mathrm{~m}$ de profundidad, que tiende a aumentar cerca del Canal Serrate. El paso desde Pan de Azúcar hacia la Isla Santa Rosa posee una longitud de $1.53 \mathrm{~km}$ con una profundidad de $15 \mathrm{~m}$ y, la distancia entre la Isla Santa Rosa y Morro Quemado es $1.65 \mathrm{~km}$ con una profundidad de $25 \mathrm{~m}$ aproximadamente (Figura 1a).

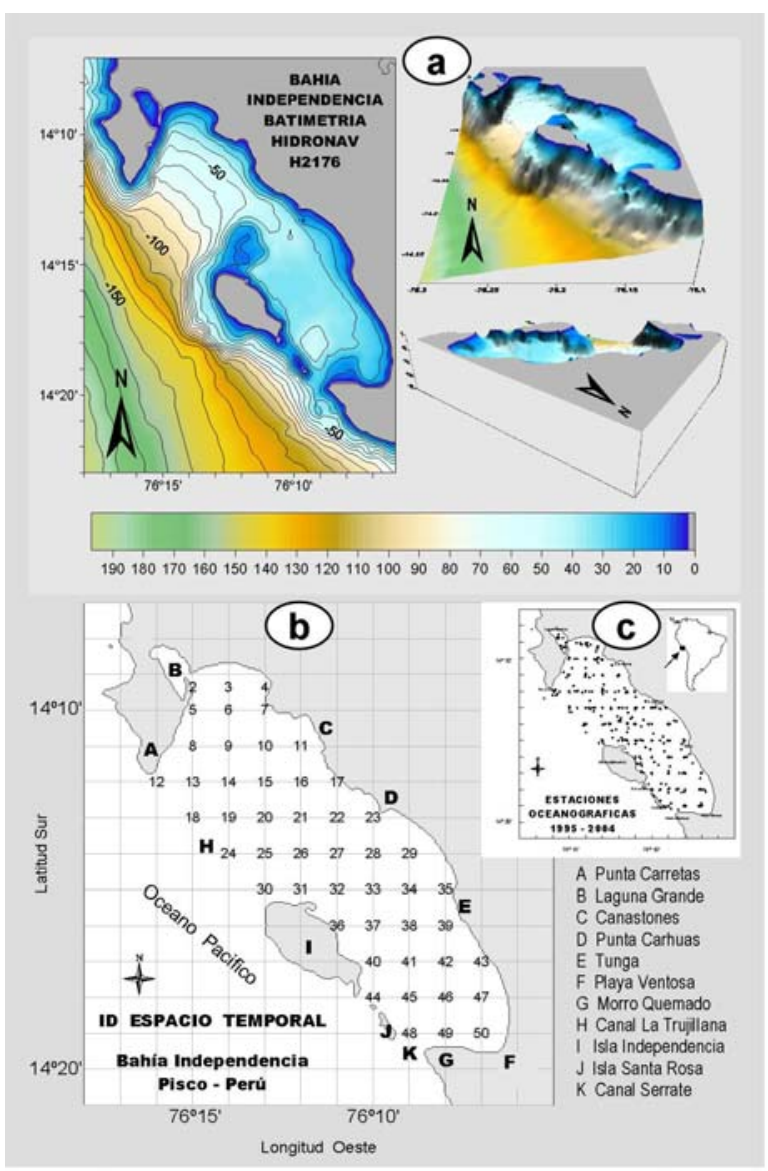

Figura 1. Ubicación y características batimétricas de Bahía Independencia. a) vista batimétrica en 2D y 3D desde diferentes ángulos. b) Ubicación de estaciones asignadas vía interpolación para el análisis espacio-temporal. c) Ubicación de estaciones registradas en prospecciones in situ durante el periodo de estudio.
Los vientos, por lo general procedentes del SE, presentan velocidades entre 5 y $10 \mathrm{~m} / \mathrm{s}$ (Morón et al., 1988). Ocasionalmente por las mañanas hay vientos del norte con velocidades moderadas de 2 a $5 \mathrm{~m} / \mathrm{s}$ (Morón, 2000). Dado que el viento ejerce tensión sobre la capa superficial del mar transmitiendo movimiento desde la superficie hacia niveles profundos (Brown et al., 2004), se torna en uno de los factores importantes que determinan las condiciones hidrodinámicas de la bahía asociados a procesos de circulación marina.

Las corrientes costeras influyen en la ubicación y magnitud de la sedimentación y la erosión (Hendry, 1993, citado en Gallegos, 2004) dando lugar a formaciones geomorfológicas relacionadas a otros factores como tipo de sedimento, variación en la energía de las olas, acción de los vientos, etc. (Cooper \& Pilkey, 2004). También influyen en la distribución de los recursos, sus huevos y larvas, así como en la localización y comportamiento de las áreas de pesca, entre otros (Gallegos, 2004). En ese contexto fueron descritos períodos cortos de circulación marina en condiciones de otoño e invierno (Sánchez et al,. 1988; Morón et al., 1988).

Las características oceanográficas conservativas de gran escala se basan en temperatura y salinidad, con las cuales se diferencian masas de agua frente a la costa peruana, descrita por diferentes autores (Zuta \& Guillén 1970, Zuta 1988, Gutiérrez et al. 2005). Estudios previos sobre la oceanografía de la bahía indican que durante El Niño 82-83 se registró una temperatura superficial máxima de $23.5{ }^{\circ} \mathrm{C}$ (Morón et al., 1988). Morón (2000) indica que las Aguas Ecuatoriales Subsuperficiales (AESS) y las Aguas Templadas de la Subantártica (ATSA) suelen mezclarse aquí por estar en una zona de afloramiento $\left(14^{\circ}-15^{\circ} \mathrm{S}\right)$.

Haciendo referencia al recurso - ambiente se han desarrollado múltiples evaluaciones biológicas, asociando su variabilidad con algunos parámetros abióticos, como los que se refieren a la composición y distribución de fitoplancton (Sánchez et al., 1988), organismos bentónicos (Jurado \& Rubio 1988, Mendo et al. 1988; Yamashiro \& Mendo 1988, Mendo et al. 1987, Mendo et al. 1989, Yamashiro et al. 1995, Rubio \& Tafur 1997, Rubio \& Taipe 1997, Zeballos 1998, Rubio et al. 2000a, Rubio et al. 2000b, Rubio et al. 2001), entre otros, llegando a asumir que la variabilidad de estos se atribuye a algunos parámetros como la circulación marina, condiciones de temperatura asociadas al fenómeno de EN, e influencia de los vientos.

Aspectos descriptivos e interpretativos de un medio físico (Palacios-Prieto et al. 2004), coadyuvan a (i) entender las condiciones óptimas en las cuales cumplen su ciclo biológico algunos recursos (Vélez 2004), (ii) advertir manifestaciones asociadas a cambios climáticos y posibles impactos ambientales 
(Gallegos 2004), (iii) inferir en algunas consecuencias de actividades antrópicas en la zona costera y, (iv) ponderar la oferta natural territorial y, cómo podría satisfacer de manera ambientalmente óptima y económicamente sustentable las demandas de un ordenamiento estatal, en el marco de acuerdos regionales y mundiales, como los establecidos en el Programa de Acción para el Desarrollo Sostenible (Palacios-Prieto et al. 2004).

En el presente estudio se integró e interpretó los datos de varias prospecciones oceanográficas del IMARPE con el objetivo de describir las características de variabilidad espacial y temporal de condiciones hidrofísicas en la bahía desde 1995 al 2004, evaluando la batimetría, vientos, circulación marina, temperatura y salinidad. La información generada es útil para entender algunos procesos naturales que ocurren en la bahía, brindando insumos de conocimiento básico para la gestión del ecosistema.

\section{Materiales y métodos.}

Datos.

Se digitalizó datos batimétricos a partir de la carta HIDRONAV H2176 (1:40000) (DHN, 1993). Se procesó datos de viento in situ registrados por la Dirección de Hidrografía y Navegación (DHN) en San Juan, sobre el litoral (75 09' 34" W, $15^{\circ} 20^{\prime} 35^{\prime \prime}$ S), disponibles entre 2001 - 2003; y de vientos estimados por satélite (QuikSCAT) obtenidos a través del Jet Propulsion Laboratory (JPL, 2008), frente a Bahía Independencia $\left(76^{\circ} 15^{\prime} \mathrm{W}, 14^{\circ} 15^{\prime} \mathrm{S}\right)$, disponibles a partir de mediados de 1999 al 2004. Para el período entre 1995 a 2004, se recopilaron datos de temperatura, salinidad, velocidad y dirección de corrientes a partir de informes internos del Instituto del Mar del Perú (IMARPE) orientados a la Evaluación de Invertebrados Marinos en Bahía Independencia (Rubio et al., 2001), cuyas prospecciones reportan una duración de 5 a 6 días. La irregular distribución espacial de los datos in situ (Figura 1c) fue escalada a una grilla regular de $0.5^{\prime}$ por 0.5’ en coordenadas geográficas, vía interpolación con el método kriging (Bresnahan \& Dickenson, 2008), generando un set de 49 estaciones identificadas espacialmente con un mismo código para los análisis (Figura 1b).

Análisis.

Las series de tiempo de los vientos de ambas fuentes fueron evaluadas en base a la componente zonal (E-W) y meridional (S-N), los periodos coincidentes fueron correlacionados usando el coeficiente de Pearson. La circulación marina fue descrita a partir de gráficos de corrientes compuestos diariamente. Se caracterizó la variabilidad de la temperatura y de la salinidad, estimando promedios, intervalos de confianza al 95\% (IC), coeficientes de variación (CV) e índices de anomalía para los niveles de superficie y fondo, las anomalías fueron relacionadas con la ubicación latitudinal usando el coeficiente de Spearman. La variabilidad espaciotemporal se representó en mapas, aplicando escalas de color basadas en el modelo de color aditivo RGB (López, 2004), distribuyendo la gama de colores de acuerdo a análisis de estadística descriptiva. Se asignó el color rojo al valor máximo, azul al mínimo, verde a la media y a la mediana, celeste al primer cuartil y amarillo al tercer cuartil. Los valores atípicos (outliers) fueron filtrados considerando como valor máximo al percentil 99 y como mínimo al percentil 1 . Los patrones de distribución espacial se diferenciaron con coeficientes de similaridad de Bray Curtis (S) definido en un rango de valores de 0 a $100 \%$ (Clarke \& Gorley, 2006), identificando grupos que fueron evaluados respecto al espacio y tiempo mediante análisis de varianza (ANOVA) de dos vías. Los grupos fueron ubicados en mapas de color asociados a la media temporal de cada estación, los parámetros fueron integrados conceptualmente.

\section{Resultados y discusión.}

Variabilidad temporal de los vientos.

En periodos coincidentes de vientos registrados in situ y satélite, la velocidad modular frente a la bahía osciló entre 1.3 a 12.9 m.s ${ }^{-1}$ con un promedio de 5.9 (IC: 5.8 - $6.1 \mathrm{~m} . \mathrm{s}^{-1}$ ), inferior a San Juan donde osciló

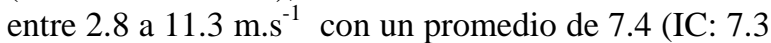
- $7.5 \mathrm{~m} . \mathrm{s}^{-1}$ ). Sin embargo los registros de viento de las dos localidades se hallan significativamente correlacionados, con valores de $r=0.39$ y $r=0.43$, para las componentes zonal y meridional respectivamente.

Los vientos frente a la bahía variaron con el ciclo estacional, con una velocidad modular promedio de $7.1 \mathrm{~m} . \mathrm{s}^{-1}$ durante el invierno y de $4.7 \mathrm{~m} . \mathrm{s}^{-1}$ durante el verano. Para otoño y primavera, las velocidades promediaron 6.3 y $5.9 \mathrm{~m} . \mathrm{s}^{-1}$ respectivamente. En la componente zonal, la velocidad promedio durante el verano fue de $-2.36 \mathrm{~m} . \mathrm{s}^{-1}$, mientras que durante el invierno alcanzó $-4.1 \mathrm{~m} . \mathrm{s}^{-1}$. En la componente meridional la velocidad promedió $4.0 \mathrm{~m} . \mathrm{s}^{-1}$ en verano y $5.7 \mathrm{~m} . \mathrm{s}^{-1}$ en invierno. Por consiguiente, en invierno los vientos fueron más intensos hacia el oeste que durante el verano (Figura 2).

\section{Circulación marina.}

La extensa plataforma de la zona sur de la bahía suele presentar flujos de circulación similares en los niveles de superficie y fondo. Desde la zona central hacia el sur ocurren movimientos en sentido horario, impulsados por aguas que ingresan a través del Canal La Trujillana con dirección hacia la zona de Carhuas, las cuales condicionadas por la configuración batimétrica, viran hacia el sur y luego hacia el oeste con dirección al Canal Serrate; luego una parte fluye hacia fuera de la bahía y la otra vira hacia la Isla 


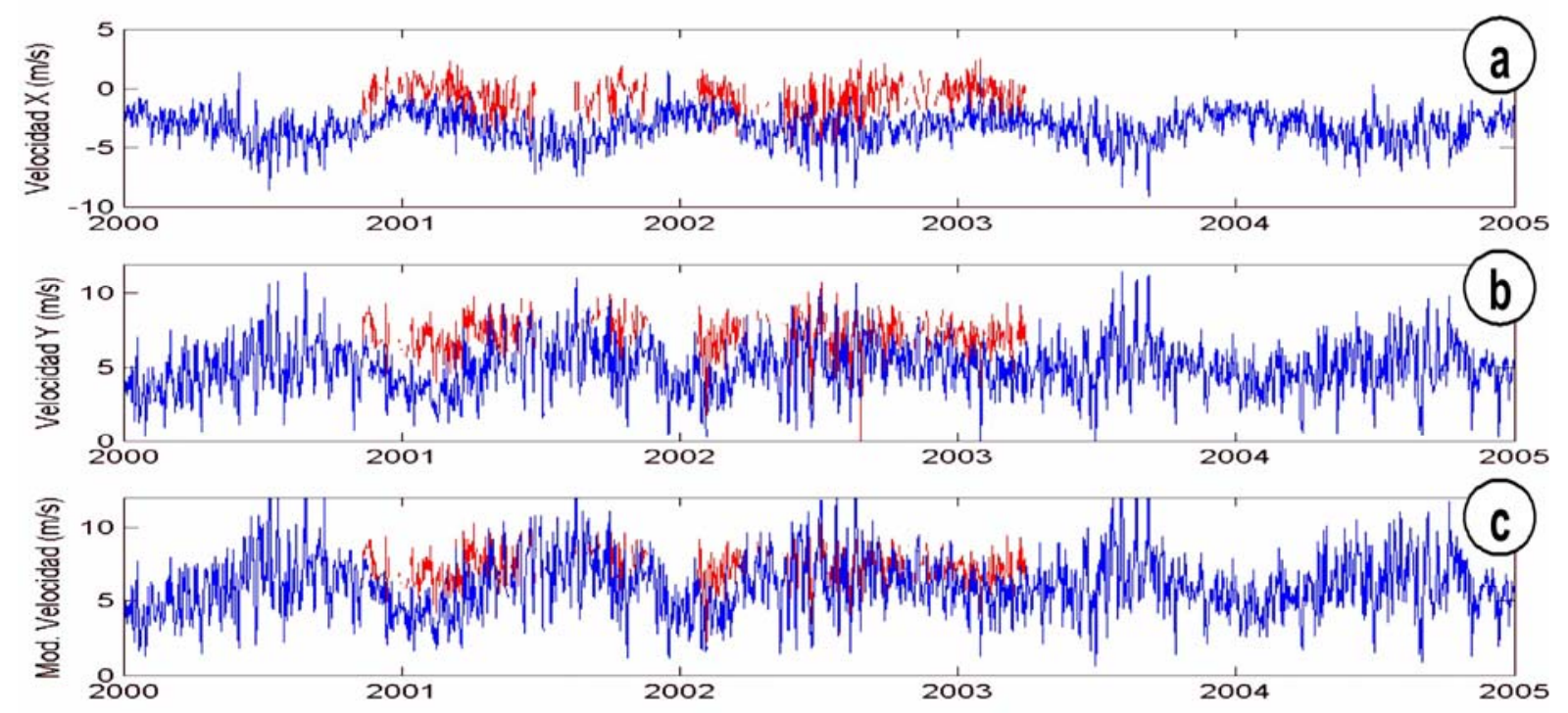

Figura.2. Comparación de velocidad del viento In Situ en San Juan de Marcona (líneas rojas) vs. viento satelital (líneas azules) frente a Bahía Independencia. a) Velocidad zonal. b) Velocidad meridional. c) Magnitud de velocidad.

Independencia con dirección al norte impulsada por aguas que ingresan por el somero paso que se halla entre las Islas Independencia y Santa Rosa, tal como se observó en el verano de 1997 (Figura 3a).

Ocasionalmente, las aguas que ingresan sobre el nivel del fondo a través del Canal La Trujillana, llegan hasta el extremo sur con tendencia a circular en sentido horario, son modificadas en su curso, cambiando el sentido de la circulación a nivel de superficie, por el efecto de las aguas que ingresan a través del Canal Serrate en dirección a las costas de Playa Ventosa, las cuales convergen con las aguas precedentes virando hacia el norte y luego hacia el oeste, generando una pequeña zona de giro antihorario frente al Canal Serrate, tal como se observó en el verano del 2002 (Figura 3c). En otro periodo como el verano de 1998 se observó que éste proceso se acentúa

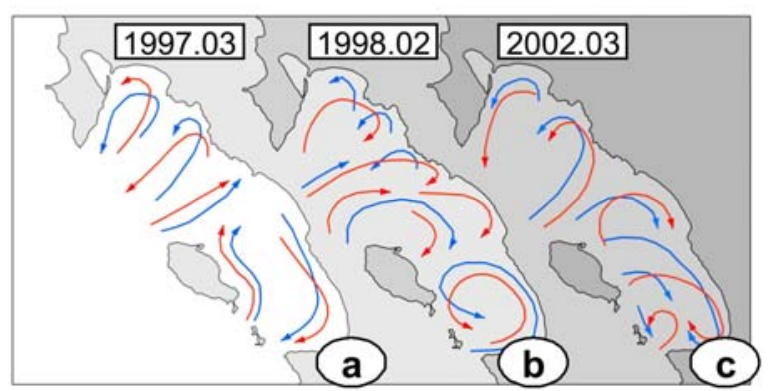

Figura 3. Diagrama conceptual de circulación marina a nivel de superficie (líneas rojas) y fondo (líneas azules), basado en la composición gráfica de vectores de corrientes. a) Verano de 1997. b) Verano de 1998. c) Verano de 2002. más logrando acoplar la circulación en ambos niveles en sentido antihorario. Espacialmente este giro no llega a extenderse más al norte de las latitudes intermedias de la Isla Independencia, porque es limitado por la incursión de aguas procedentes del Canal La Trujillana (Figura 3b).

Las aguas que incursionan a través del Canal La Trujillana sobre el fondo hacia Carhuas, Canastones y norte de la bahía, al llegar a zonas de baja profundidad tienden a cambiar el sentido de su circulación describiendo un movimiento antihorario, las cuales ya en el nivel de superficie proyectan flujos de salida hacia el Canal La Trujillana, evidenciando un proceso de afloramiento con ciertas características tipo Ekman (Brown et al., 2004), tal como se observó en las prospecciones de verano de 1997 y 2002. En 1998, este patrón se vio modificado a nivel de superficie, ya que los flujos de ingreso a través del Canal La Trujillana hacia la zona norte de la bahía, Canastones y Carhuas, tendieron a proyectar flujos de retorno en sentido horario, sin afectar el nivel de fondo.

Variabilidad espacio temporal de temperatura.

La temperatura superficial del mar (TSM) mostró un rango de mayor variabilidad espacial (CV: 3.7 $12.9 \%)$ que la temperatura de fondo (TFM) (CV: 1.8 $3.5 \%)$. Los rangos de temperatura se incrementaron en verano y se redujeron en periodos asociados a la incursión de aguas con características homogéneas que llegan a cubrir toda la bahía, como ocurrió en el verano de 1998 (EN) y en el otoño de 2001. En ambos niveles la variación temporal es significativa $(\mathrm{p}<0.05)$. En superficie los promedios de temperatura oscilaron entre 15.8 (IC: $15.5-16{ }^{\circ} \mathrm{C}$ ) y $23.5^{\circ} \mathrm{C}$ (IC: 23.2 - 23.8 ${ }^{\circ} \mathrm{C}$ ) (Figura 4a). A nivel de fondo las temperaturas 
promedio oscilaron entre 13.8 (IC: 13.5 - $14.1{ }^{\circ} \mathrm{C}$ ) y $21.8^{\circ} \mathrm{C}$ (IC: $21.4-22.1^{\circ} \mathrm{C}$ ) (Figura 4b).

En 6 de las 10 prospecciones las temperaturas en superficie $y$ en fondo presentaron correlación significativa: 1995-03 ( $\mathrm{r}=0.30), 1996-04(\mathrm{r}=0.45)$, 1997-03 ( $\mathrm{r}=0.30), 2001-05(\mathrm{r}=0.31), 2002-03(\mathrm{r}=$ $0.40)$ y 2003-04 ( $\mathrm{r}=0.50)$, evidenciando patrones en la distribución espacial de temperatura que asocia a ambos niveles, en cambio en períodos como la primavera de 1998 y los veranos de 1998, 1999 y 2004, la distribución espacial difiere entre ambos niveles.

La similaridad de la distribución espacial de TSM es menor que la TFM, en superficie se identificaron 4 grupos $(S=88.5)$, constituido por “a” = 7 , “b” = 15 , “c” $=6$, “d” $=21$ estaciones (Figura 5a). En fondo se identificaron 2 grupos con un coeficiente de $\mathrm{S}=99.2$ constituido por "a" = 14 y "b" = 35 estaciones (Figura 5b). En cada nivel los grupos identificados difieren significativamente entre sí como a través del tiempo $(\mathrm{p}<0.05)$.
La distribución espacial de estos grupos en superficie describen un gradiente latitudinal caracterizado por temperaturas altas al norte de la bahía y bajas en la zona sur (Figura 5e). Las estaciones con mayor temperatura corresponden al grupo "b": en condiciones normales la TSM osciló entre 16.8 a $21.2{ }^{\circ} \mathrm{C}$, alcanzando $24.3{ }^{\circ} \mathrm{C}$ (IC: 24.0 $24.6{ }^{\circ} \mathrm{C}$ ) al incluir el verano de 1998 (EN). Las estaciones de menor temperatura corresponden al grupo "d"; aquí la TSM en condiciones normales estuvo entre 14.4 a $19.2^{\circ} \mathrm{C}$, e incluyendo el verano de 1998 (EN) llegó hasta $22.7^{\circ} \mathrm{C}$ (IC: 22.4 - $22.9^{\circ} \mathrm{C}$ ) (Figura 5c). Las distribución de la TSM puede deberse al repliegue de aguas en la zona norte por acción del viento, favoreciendo al incremento de temperaturas por efecto constante de la radiación solar y el contacto con aguas con mayor temperatura procedentes del área semi-encerrada de Laguna Grande.

En el fondo, las estaciones de mayor temperatura son de menor profundidad, corresponden al grupo "a" y se ubican próximas a las costas norte y centro de la

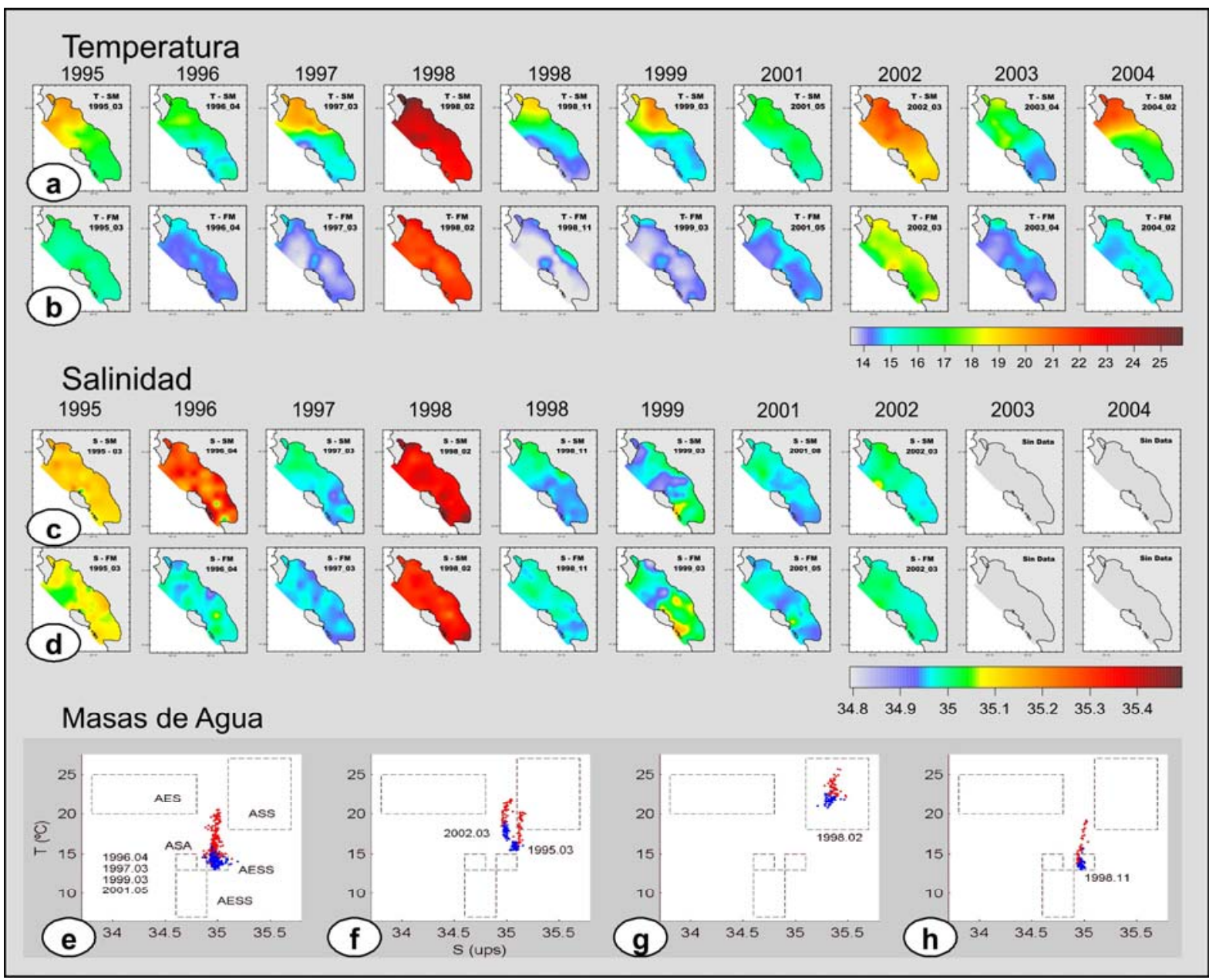

Figura 4. Variabilidad espacio-temporal de Temperatura y Salinidad e identificación de masas de agua con diagramas T-S, diferenciando el nivel de superficie (puntos rojos) y fondo (puntos azules) en diferentes periodos dentro de Bahía Independencia. a) Temperatura en superficie. b) Temperatura en fondo. c) Salinidad en superficie. d) Salinidad en fondo. e) Periodos normales con AESS. f) Periodos normales con aguas de mezcla. g) Período El Niño con ASS. h) Periodo La Niña con AESS. 
bahía, así como en la zona de la pampa (Figura5f), cuyos promedios en condiciones normales oscilaron entre 14.8 a $18.3^{\circ} \mathrm{C}$ e incluyendo el verano de 1998 (EN) alcanzaron hasta $22.2^{\circ} \mathrm{C}\left(\mathrm{IC}: 22.1-22.7^{\circ} \mathrm{C}\right)$. En estaciones de mayor profundidad, correspondientes al grupo "b", las temperaturas promedio normalmente oscilaron entre 13.6 a $17.6{ }^{\circ} \mathrm{C}$ y considerando el verano de 1998 llegaron hasta $21.6^{\circ} \mathrm{C}$ (IC: 21.9 - 21.8 $\left.{ }^{\circ} \mathrm{C}\right)$ (Figura 5d).

(a)

TSM- SIMLARIDAD DE ESTACIONES - B. NDEPENDENCL - PISCO
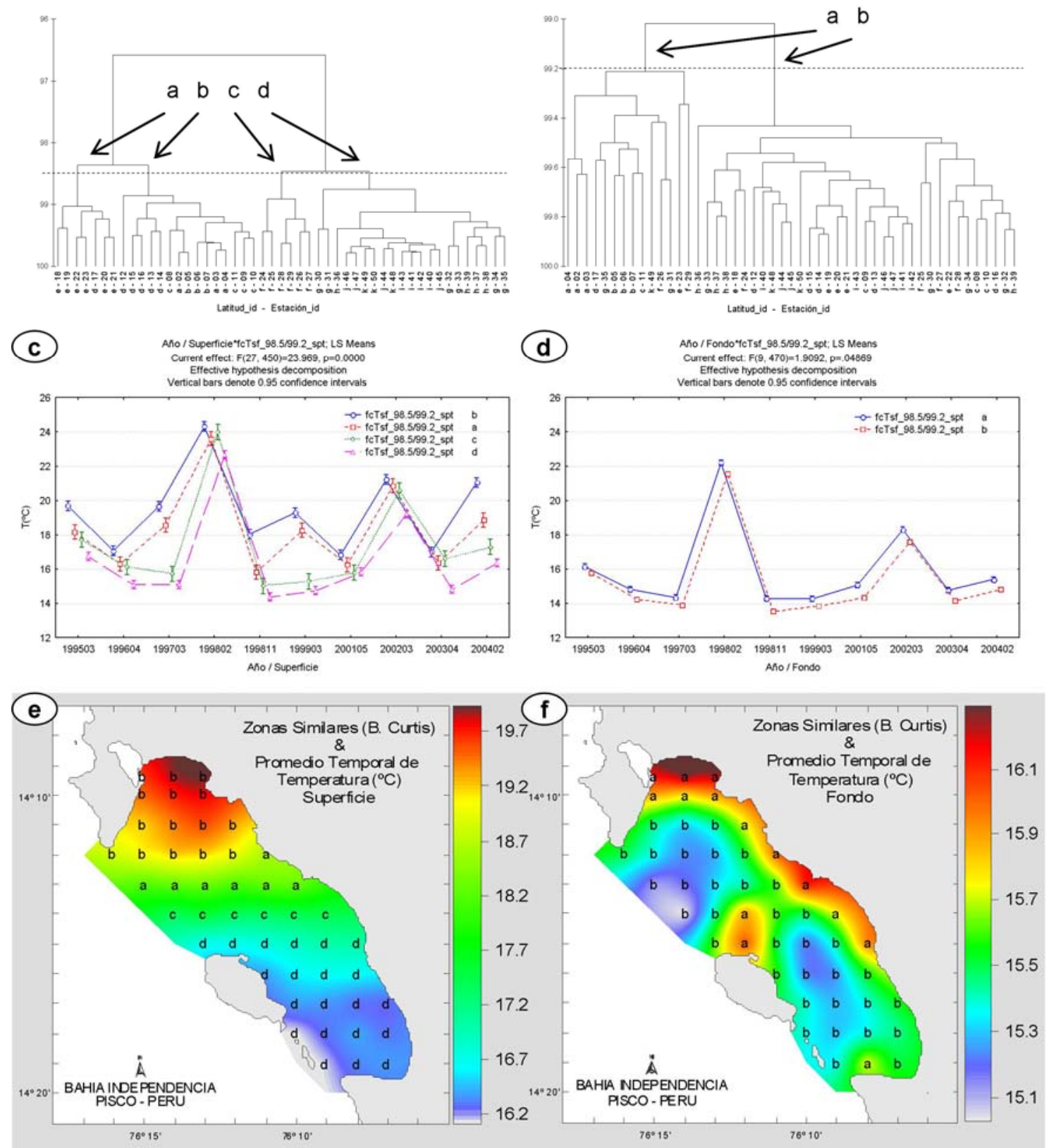

Figura 5. Patrones de variabilidad espacio-temporal de temperatura determinados por análisis de similaridad, variación espacio temporal de grupos clasificados y ubicación espacial asociada al promedio temporal, en mapas de color. a) Agrupamientos de temperatura en superficie y b) en fondo; c) análisis de varianza de los grupos en superficie y d) en fondo; e) ubicación espacial de grupos en superficie y f) en fondo. 
oscilaron desde 34.95 (IC: 34.94 - 34.96 ups) hasta 35.33 ups (IC: 35.32 - 35.34 ups), que corresponden a las prospecciones del verano de 1997 y el verano de 1998 respectivamente (Figura 4d). Resaltan incrementos generalizados de la salinidad en toda la bahía en las prospecciones de los veranos de 1995 y 1998, asociadas a la incursión de Aguas Subtropicales Superficiales.
Dado que el rango de variabilidad es bajo, las distribuciones de salinidad en ambos niveles se correlacionan poco, siendo la correlación significativa sólo en 2 de las 8 prospecciones, que corresponden a la primavera de $1998(\mathrm{r}=0.46)$ y verano del $2002(\mathrm{r}=$ 0.39). En ambas la distribución a ambos niveles se caracterizó por un ligero gradiente latitudinal con salinidades altas en el norte de la bahía y bajas en la (a)

SSM - SMLARDAd DE Estaciones - B. NDEPENDENCH - PSCO

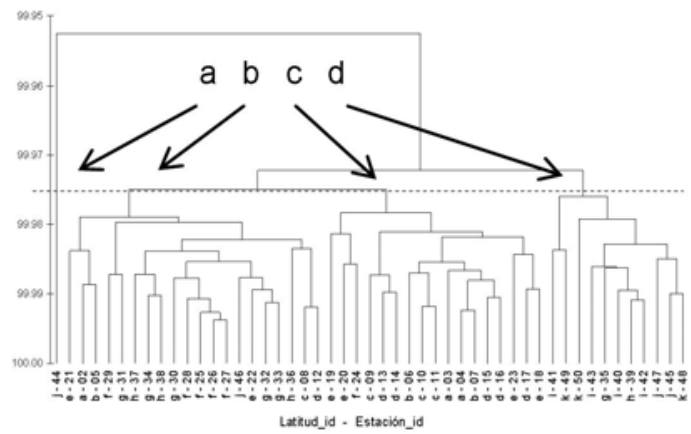

(c)
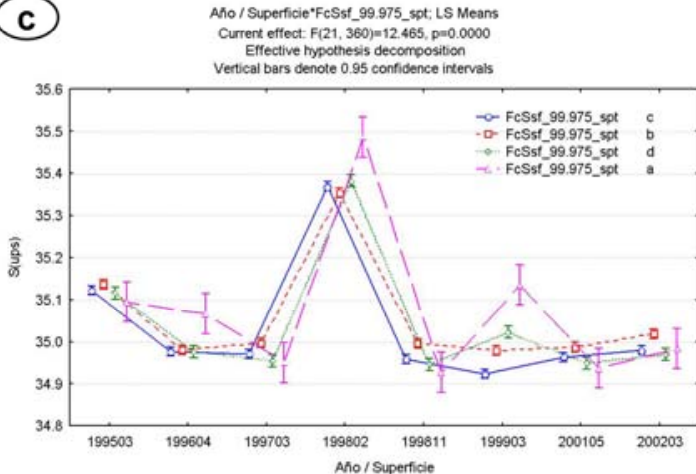

(b)

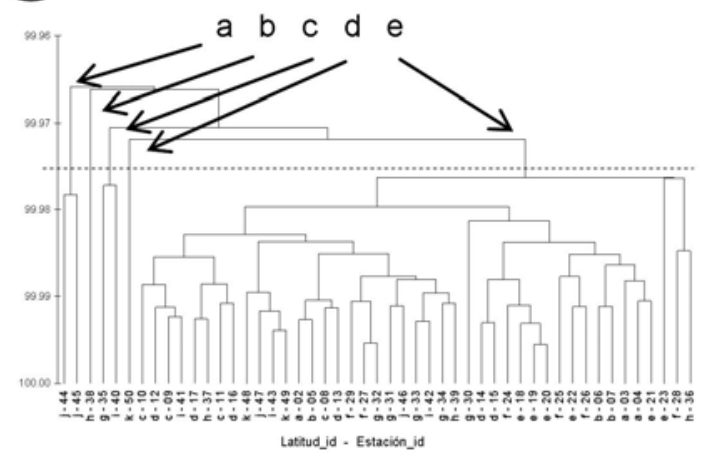

(d)

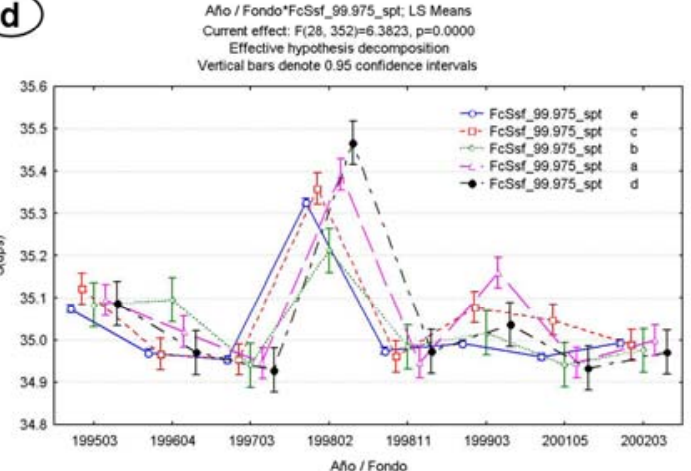

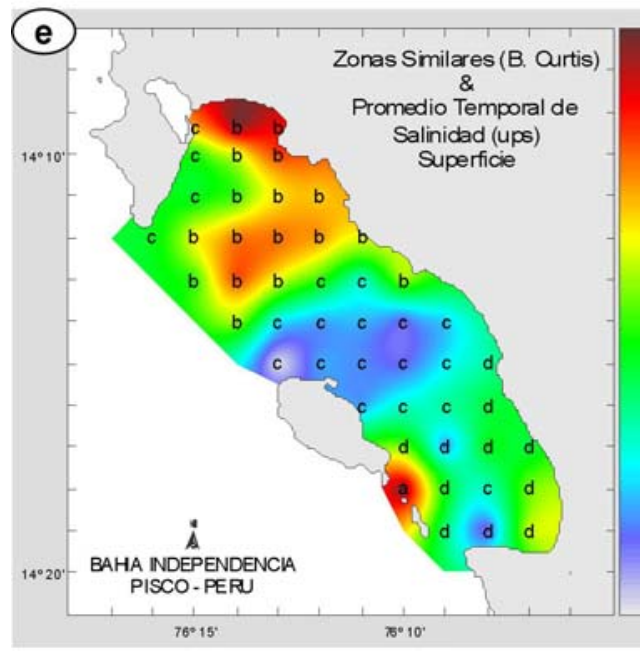
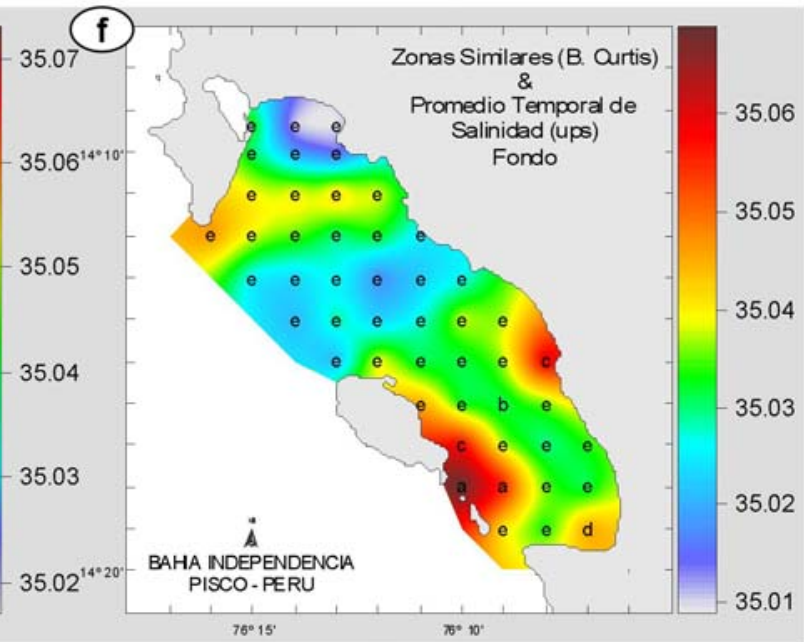

Figura 6. Patrones de variabilidad espacio-temporal de salinidad determinados por análisis de similaridad, variación espacio temporal de grupos clasificados y ubicación espacial asociada al promedio temporal, en mapas de color. a) Agrupamientos de salinidad en superficie y b) en fondo; c) análisis de varianza de los grupos en superficie y d) en fondo; e) ubicación espacial de grupos en superficie y f) en fondo. 
zona sur.

En la distribución espacial de salinidad se identificó grupos de estaciones para ambos niveles que difieren significativamente entre sí a través del tiempo $(\mathrm{p}<0.05)$. En superficie se identificó 4 grupos ( $\mathrm{S}=99.975)$, constituidos por “a” = 1, "b” = 17, “c” = 20 y "d" = 11 estaciones (Figura 6a), mientras que en el fondo con el mismo coeficiente, se identificó 5 grupos, constituidos por "a" = 2, "b" = 1 , “c" = 2, "d" $=1 \mathrm{y}$ " $\mathrm{e}$ " $=43$ estaciones (Figura 6b).

La distribución espacial de estos grupos se refleja en parte en los mapas de promedio temporal. En superficie las estaciones de mayor salinidad se hallaron al norte de la bahía frente a Laguna Grande, Canastones y extendiéndose hacia el centro del Canal la Trujillana, (grupo “b”), y en otra pequeña zona ubicada al sur-este de la Isla Independencia ("a”) (Figura 6e). En el resto de la bahía los valores de salinidad fueron menores (Figura 6c). A nivel de fondo se hallaron pequeñas zonas de mayor salinidad al sur de la Isla Independencia (“a”), frente a Playa Ventosa (“d”) y, frente a Tunga (“c”) (Figura 6f). En el resto de la bahía predominaron bajas salinidades, que en condiciones normales osciló entre 34.95 35.07 ups y se incrementó hasta 35.33 ups en condiciones EN (Figura 6d).

La distribución espacial de salinidad se asocia congruentemente con la circulación en la bahía, evidenciada por zonas que presentan características que difieren de sus alrededores, como las que se observa al sur-este de la Isla Independencia, donde la salinidad es similar en ambos niveles, manifestándose con valores altos; en el nivel de fondo es notorio frente a Playa Ventosa la cual es favorecida por la forma cóncava de sus costas y, frente a Tunga que estarían asociadas a una zona de confluencia de aguas procedentes de la zona norte y sur de la bahía (Figuras 3a, b). Al norte de la bahía, frente a Laguna Grande las condiciones son particulares, en superficie se observa salinidades altas como efecto del repliegue de aguas por acción de los vientos y las condiciones locales de Laguna Grande, mientras que a nivel de fondo difieren de superficie por ser de menor salinidad, el cual estaría asociado a aguas afloradas que aún no han tenido influencia del nivel de superficie (Figuras 6e, f), algunos periodos particulares se observa en los otoños de 1996 y 2001 (Figuras 4c, d)

Masas de agua.

En relación a las fases del ciclo ENOS, las AESS se presentaron en la bahía tanto en condiciones neutras (otoños de 1996, 2001 y veranos de 1997 y 1999, Fig. 3e), como en condiciones de La Niña (primavera de 1998, Figura 3h). En las condiciones de El Niño del verano de 1998, incursionaron Aguas Subtropicales Superficiales (ASS; Figura 3g).

Solo en dos de los periodos de estudio se observó aguas de mezcla con influencia de las ASS, cuyas características se diferenciaron claramente desde el nivel de fondo, como se observó en el verano de 2002 y al verano de 1995; en éste último período los valores de salinidad aún se aproximan al rango de las ASS, pero ya con bajas temperaturas (Figura $3 f$ ).

Cabe señalar que se puso mayor atención en las características del nivel de fondo para identificar las masas de agua, de acuerdo a rangos descritos (Zuta \& Guillén 1970, Zuta 1988, Gutiérrez et al. 2005), ya que conservan mejor las propiedades físicas de temperatura y salinidad, frente a los efectos que interactúan en el nivel de superficie

\section{Conclusiones.}

La interacción de vientos y circulación marina genera condiciones favorables a procesos de afloramiento en la zona norte da la bahía, ya que las aguas que ingresan por el norte de la bahía través del Canal La Trujillana, tienden a retornar hacia el mismo canal por el nivel de superficie, mientras que en la zona sur la circulación proyecta giros de gran extensión que en el nivel de fondo favorecerían a conservar aguas con tiempo de residencia mayor que en la zona norte.

El patrón de distribución espacial de temperatura y salinidad, se debe a procesos de escala local, asociados a la interacción de vientos, circulación marina y el entorno geomorfológico de la bahía. A nivel de superficie se generan gradientes latitudinales decrecientes de norte a sur en salinidad y especialmente en temperatura.

Los patrones de distribución espacial que se generan con mayor frecuencia mientras permanecen las AESS, logran ser alterados por el efecto de variabilidad temporal a mayor escala en algunos periodos, debido a la incursión de aguas de mezcla o de ASS en condiciones El Niño extremas.

\section{Agradecimientos.}

Se agradece al personal del Instituto del Mar del Perú que participó en el inicio, conducción y culminación de este informe. El estudio se condujo en el marco del proyecto CENSOR (Climate variability and El Niño Southern Oscillation: Implications for natural coastal resources and management), de la UE, contrato 511071.

\section{Literatura citada.}

Argüelles J., Morón O., Chang F. \& Lorenzo A. 1999. Evaluación de la población de concha de abanico (Argopecten purpuratus) en la Bahía Independencia, Pisco, Perú 12 al 25 de febrero de 1998. Inf. Interno. 47 pp. Inst. Mar Perú. Callao.

Bresnahan T. \& Dickenson K. 2008. Surfer 8 Self-Paced Training Guide. Technical Support. Golden Software, Inc.

Brown E., Colling A., Park D., Phillips J., Rothery D. \& Wright J. 2004. Ocean Circulation. Open university course team. Butterworth-Heinnemann, Oxford. 
Clarke K. \& Gorley R. 2006. PRIMER v6 User manual/tutorial, PRIMER-E, Plymouth UK.

Cooper J.A.G. \& Pilkey O.H. 2004. Sea-level rise and shoreline retreat: time to abandon the Bruun Rule. Global and Planetary Change. 43 (2007): 157-171.

Dirección de Hidrografía y Navegación. 1993. Atlas hidrográfico del Perú. $2^{\text {a }}$ Edición. Callao.

Gallegos A. 2004. Clima oceánico: los mares mexicanos ante el cambio climático global. En: Martinez J. Fernandez A. (Eds). Cambio climático una visión desde México. D.R. INE, México, ISBN 968-817-704-0. : 4151.

Gutiérrez D., Arones K., Chang F., Quipuzcoa L. \& Villanueva P. 2005. Impacto de la variación oceanográfica estacional e interanual sobre los ensambles de microfitoplancton, mesozooplancton, ictioplancton y macrozoobentos de dos áreas costeras del norte del Perú entre 1994 y 2002. Bol Inst Mar Perú, - Callao. Vol. 22, Nos. 1 y 2.

Jet Propulsion Laboratory. 2008. Winds. Measuring winds from space. On-line: http://winds.jpl.nasa.gov/.

Jurado E. \& Rubio J. 1988. Algunas relaciones biométricas de la concha de abanico (Argopecten purpuratus) de la bahía Independencia, Perú. Bol Inst Mar Perù, Vol. Ext.: 169 - 171.

López $\quad$ F. 2004.2 ColorSynk. fernando@macprogramadores.org.

Mendo J. \& Wolff M. 2003. El impacto de El Niño sobre la producción de Concha de Abanico (Argopecten purpuratus) en Bahía Independencia, Pisco, Peru. Ecol.Apl. 2(1): 51-57.

Mendo J., Valdivieso V. \& Yamashiro C. 1988. Cambios en densidad, número y biomasa de la población de concha de abanico (Argopecten purpuratus) en la Bahía Independencia (Pisco, Perú) durante 1984 - 1987. Bol. Inst. Mar Perú - Callao Vol. Extraodinario.: 153-162.

Mendo J., Valdivieso V., Yamashiro C., Jurado E., Morón O. \& Rubio J. 1987. Evaluación de la población de concha de abanico (Argopecten purpuratus) en la bahía Independencia, Pisco, Perú, 17 de enero - 4 de febrero de 1987. Inf. Inst. Mar Perú. IMARPE: 91- 64.

Mendo J., Yamashiro C., Rubio J., Kameya A., Jurado E., Maldonado M. \& Guzman S. 1989.. "Evaluación de la población de concha de abanico (Argopecten purpuratus) en la bahía Independencia, Pisco Perú, 23 de setiembre al 09 de octubre de 1987.” Inf. Inst. Mar Perú 94-95: 65.

Morón O., Lostanau N. \& Escudero L. 1988. Parámetros oceanográficos en Bahía Independencia, Perú, entre mayo de 1985 y julio de 1987. Bol.Inst. Mar Perú Callao Vol. Extraodinario.: 25-34.

Morón O. 2000. Características del ambiente marino frente a la costa peruana. Bol Inst. Mar Perú. 19(1-2): 179-204.

NOAA. 2007. Cold and warm episodes by season. On-line: www.cpc.ncep.noaa.gov/ products/analysis_monitoring/ensostuff/ensoyears.shtml.

Orrego H. 2002. Estudio básico sobre las condiciones físicas, oceanográficas y ambientales de Laguna Grande (Pisco) durante 1999 - 2001.: 15-23. En: Mendo \& Wolf (editores) Memorias I Jornada Científica "Bases ecológicas y socioeconómicas para el manejo de los recursos vivos de la Reserva Nacional de Paracas”. Lima - Perú.
Palacios-Prieto J.L., Sanchez-Salazar M.T., Casado J.M., Propin E., Delgado J., Velazquez A., Chias L., Ortiz M.I., Gonzáles J., Negrete G., Gabriel J., Marquez R., Nieda T., Jimenez R., Muñoz E., Ocaña D., Juarez E., Anzaldo C., Hernández J.C., Valderrama K., Rodriguez J., Campos J.M., Vera H. \& Camacho C.G. 2004. Indicadores para la caracterización y el ordenamiento territorial. UNAM, SEDESOL, SEMARNAT, INE, México, ISBN 970-32-1885-7. pp. 161.

Perlin A., Moum J.N., Klimak J.M., Levine M.D., Boyd T. \& Kosro P.M. 2007. Organization of stratification, turbulence, and veering in bottom Ekman layers. J. Geoph. Res. 112. pp 12.

Ramos E., Tarazona J., Indacochea A. \& Valle S. 1999. Asentamiento larval de invertebrados marinos bentónicos en Bahía Independencia Pisco - Perú antes, durante y después de "El Niño 1997-98". VIII Congreso Latinoamericano sobre Ciencias del Mar, Trujillo Perú: 760-761.

Rubio J., Galindo O., Flores D. \& Quispe S. 2001 Evaluación de la almeja (Gari solida), en Bahía Independencia, Pisco, Inf. Prog. 151, 12 pp Inst Mar Perú - Callao.

Rubio J., Aguilar S., Soto M. \& Taipe A. 2000. Evaluación poblacional del recurso concha de abanico (A. purpuratus) en Bahía Independencia del 17 al 30 de mayo 1999, Inf. Prog. 120, 16 pp Inst. Mar Perú Callao.

Rubio J., Taipe A., Castillo G., Aguilar S., Flores D., Lorenzo A., Morón O. \& Delgado E. 2000. Evaluación poblacional del recurso concha de abanico (Argopecten purpuratus) en Bahía Independencia, Pisco, del 04 al 18 de marzo de 1999”. Inf. Prog. Inst. Mar Perú $\mathrm{N}^{\circ}$ 124. Callao, IMARPE.: 36.

Rubio J. \& Tafur R. 1997. Evaluación poblacional del recurso concha de abanico (Argopecten purpuratus) en bahía Independencia. 18 de abril al 02 de mayo de 1996”. Inf. Prog. Inst. Mar Perú $\mathrm{N}^{\mathrm{o}}$ 60. Callao, IMARPE.: 11-31.

Rubio J. \& Taype A. 1997. Evaluación poblacional del recurso concha de abanico A. purpuratus en Bahía Independencia, Pisco (10 - 24 de marzo de 1997) Inf. Prog. 63: 37-51, Inst. Mar Perú - Callao.

Rubio J., Taipe A., Morón O. \& Delgado E. 1998. Evaluación poblacional del recurso concha de abanico (A. purpuratus) en Bahía Independencia, 10 - 24 de marzo de 1997. Inf. Interno. 44 pp. Inst. Mar Perú. Callao.

Sanchez S., Tarazona J., Flores R., Maldonado M. \& Carbajal G. 1988. Características del Fitoplancton de Invierno en Bahía Independencia, Perú. Bol.Inst. Mar Perú - Callao Vol. Extraodinario.: 53-64.

Segura M., Galindo O. \& Zeballos J. 1998. Monitoreo del recurso almeja (Gari solida) en Bahía Independencia. Inf. Prog. 96. 23 pp Inst. Mar Perú - Callao.

Tenorio J., Vásquez L. \& Vásquez C. 2002. Evaluación de la población del recurso concha de abanico (Argopecten purpuratus) en Bahía Independencia - Pisco 2002-03 (25 - 31 Marzo del 2002). Inf. Interno. 10 pp. Inst. Mar Peru. Callao.

Torrence Ch. \& Compo G. 1998. A practical guide to wavelet analysis. Bull. Amer. Met. Soc. 79: 61-69.

Vélez J. 2004. Ecology of larval fishes in the Independencia Bay, Pisco, Perú: temporal and spatial relationships, 
taxonomic aspects, Alfred Wegener Institute for Polar and Marine Research, University of Bremen.

Yamashiro C., Zeballos J., Rabi M., Morón O. \& Taype A. 1995. Situación del recurso concha de abanico en el área de Pisco y evaluación de la población en Bahía Independencia (18 de Marzo al 06 de Abril de 1995), Inf. Prog. 6, Inst Mar Perú-Callao.

Yamashiro C., Rubio J., Jurado E., Auza E., Maldonado M., Ayon P. \& Antonietti E. 1990. Evaluación de la población de concha de abanico (Argopecten purpuratus) en la bahía Independencia, Pisco, Perú, 20 de febrero - 04 de marzo de 1988, Inf. Inst Mar Perú. 98.
Yamashiro C. \& Mendo J. 1988. Crecimiento de la Concha de Abanico (Argopecten purpuratus) en la Bahía Independencia, Pisco, Perú. Bol. Inst. Mar Perú - Callao Vol. Extraodinario.: 163-168.

Zeballos J. 1998. Monitoreo del recurso "almeja" (Gari solida) en Bahía Independencia. Inf. Prog. Inst. Mar Perú No 96. Callao, IMARPE.: 23.

Zuta S. \& Guillén O. 1970. Oceanografía de las aguas costeras del Perú. Bol. Inst. Mar Perú - Callao. 2 (5): 157-324.

Zuta S. 1988. Variations of the mass field and currents off the Peru coast. Time Series of Ocean Measurements, Volume 4. IOC Tech. Ser. 33.

${ }^{1}$ Centro de Investigaciones en Modelado Oceanográfico y Biológico Pesquero (CIMOBP), Instituto del Mar del Perú, Esq. Gamarra y Gral. Valle, Chuchito, Callao, Perú. dquispe@imarpe.gob.pe.

2 Dirección de Investigaciones Oceanográficas (DIO), Instituto del Mar del Perú, Esq. Gamarra y Gral. Valle, Chuchito, Callao, Perú. mgraco@imarpe.gob.pe.

${ }^{3}$ Centro de Investigaciones en Modelado Oceanográfico y Biológico Pesquero (CIMOBP), Instituto del Mar del Perú, Esq. Gamarra y Gral. Valle, Chuchito, Callao, Perú. dcorrea@imarpe.gob.pe.

${ }^{4}$ Centro de Investigaciones en Modelado Oceanográfico y Biológico Pesquero (CIMOBP), Instituto del Mar del Perú, Esq. Gamarra y Gral. Valle, Chuchito, Callao, Perú. jtam@imarpe.gob.pe.

${ }^{5}$ Dirección de Investigaciones Oceanográficas (DIO), Instituto del Mar del Perú, Esq. Gamarra y Gral. Valle, Chuchito, Callao, Perú. dgutierrez@imarpe.gob.pe.

${ }^{6}$ Dirección de Investigaciones Oceanográficas (DIO), Instituto del Mar del Perú, Esq. Gamarra y Gral. Valle, Chuchito, Callao, Perú. omoron@imarpe.gob.pe.

${ }^{7}$ Dirección de Investigaciones Oceanográficas (DIO), Instituto del Mar del Perú, Esq. Gamarra y Gral. Valle, Chuchito, Callao, Perú. gflores@imarpe.gob.pe.

${ }^{8}$ Dirección de Investigaciones de Recursos Demersales y Litorales (DIRDL), Instituto del Mar del Perú, Esq. Gamarra y Gral. Valle, Chuchito, Callao, Perú. cyamashiro@imarpe.gob.pe. 